\title{
Low Frequency Oscillations and Bifurcation Diagram in Semi-Insulating GaAs Samples
}

\author{
R. L. da Silva ${ }^{1}$, H. A. Albuquerque ${ }^{1}$, R. M. Rubinger ${ }^{2}$, A. G. de Oliveira ${ }^{1}$, G. M. Ribeiro ${ }^{1}$, and W. N. Rodrigues ${ }^{1}$ \\ ${ }^{1}$ Departamento de Física, Instituto de Ciências Exatas, Universidade Federal de Minas Gerais, \\ Caixa Postal 702, 30123-970, Belo Horizonte, MG, Brazil \\ ${ }^{2}$ Departamento de Física e Química, Instituto de Ciências, \\ Universidade Federal de Itajubá, Caixa Postal 50, 37500-903, Itajubá, MG, Brazil
}

\author{
Received on 4 April, 2005
}

\begin{abstract}
We present an experimental study of bifurcation diagrams from low frequency current oscillations (LFO) measurements obtained from semi-insulating GaAs samples grown by low temperature molecular beam Epitaxy (LTMBE). The considered growth temperatures were $215^{\circ} \mathrm{C}$ and $265^{\circ} \mathrm{C}$. LFO are considered to be spontaneously generated oscillations under constant applied bias $V$. These oscillations were measurement and recorded in the form of time series. The bifurcation diagrams were obtained from the sequence of minima as a function of the applied bias. The standard measurement procedure was described elsewhere. As the control parameter, the bias allows the identification of a bifurcation route to chaos.
\end{abstract}

Keywords: Low frequency current oscillations; GaAs; Bifurcation diagrams

\section{INTRODUCTION}

Semiconductors are appropriate materials for understanding nonlinear dynamics phenomena because electrical currents, field, and other quantities can be performed under precisely controlled conditions [1-3]. The semi-insulating (SI) GaAs samples grown by low temperature (LT) molecular beam epitaxy (MBE) present a high density of As anti-site defects (around $10^{19} \mathrm{~cm}^{-3}$ ). Under extremely varied experimental conditions, such as: temperature, infrared illumination, applied bias, etc; these spontaneous currents oscillations under controlled conditions present routes to chaos.

Low frequency oscillations (LFO) are characterized by a frequency range from few $\mathrm{Hz}$ to $\mathrm{kHz}$. They occur inside a device and constitute self-organized spatio-temporal structures of electric field domains moving from cathode to anode. [1] These oscillations are easy to be observed and measured as nonlinear time series without the need of sophisticated instruments. In semiconductors presenting negative differential conductivity (NDC), moving high-field domains can build up spontaneously leading to current oscillations. In semi-insulating GaAs such oscillations occur due to the effect of field enhanced trapping of carriers been captured into deep traps. A route to find the microscopic causes of the LFO is to investigate the carrier generation and recombination processes. In previous works $[2,4]$ using static measurements we presented results that could account for the dominant generation and recombination (g-r) processes. They are, respectively, impact ionization and field-enhanced trapping. [2,4] Both processes present characteristics of avalanche and thus are critically dependent on the residual (i.e. obtained at low electric fields or at the ohmic regime) free carrier density.

In a previous work [1], we carried out analysis on experimental LFO, measured in semi-insulating GaAs samples. Those analysis were carried out considering methods of nonlinear dynamics analysis of time series and of noise reduction. $[5,6]$

In the present work, we observe LFO from semi-insulating
GaAs samples, grown to lower temperature than the previous works. [7] The bifurcation diagrams were obtained from the minima sequence of experimental time series as a function of the applied bias. A global view of the underlying dynamics is presented through bifurcation diagrams which are convenient to investigate the periodicity and to classify the bifurcations of the current oscillations versus a control parameter.

\section{EXPERIMENTAL DETAILS}

The semi-insulating GaAs samples were grown (LT-MBE). Two samples were grown, one at $215^{\circ} \mathrm{C}$ and the other at $265^{\circ} \mathrm{C}$. At these growth temperatures GaAs samples becomes non-stechiometric and incorporates a high density of deep level defects. An active layer of $2.3 \mu \mathrm{m}$ thick was grown on top of a liquid encapsulated Czochralski GaAs wafer. Bar shaped pieces were cut from these GaAs crystals. Typical sample dimensions were $2.0 \mathrm{~mm}$ length and $1.0 \mathrm{~mm}$ width. Two parallel indium stripes $0.5 \mathrm{~mm}$ apart were deposited as ohmic contacts. The measurements were carried out in a cold-finger liquid He cryostat with temperature control. A commercial infrared GaAs LED placed inside the cryostat illuminated the samples. A Keithley 237 voltage source was used as the bias and a National Instruments data acquisition board (DAQ) was used to record data time series in a personal computer. Even though the measuring circuit being quite simple, for semi-insulating samples as ours, it is necessary to provide an experimental setup with external noise rejection (mainly random noise and common mode at $60 \mathrm{~Hz}$ ). For this we considered a field-effect transistor near the sample in a source-follower configuration. The intrinsic noise generated by the sample itself, cannot be avoided and in this case, the best solution is to apply a dynamical filter, commonly known as a noise reduction algorithm which is proper to the analysis of time series due to its property of not deforming signal and its phase. [1,6-8] 


\section{EXPERIMENTAL RESULTS}

The first sample will be addressed as BH9816 and is the one grown at $215^{\circ} \mathrm{C}$. Fig. 1 show the I(V) characteristics for measurements at $160 \mathrm{~K}$ under illumination with a constant current of $15 \mathrm{~mA}$ through the LED. The curve was traced using an in series circuit consisting of a DC voltage source, the sample and a resistor. For each fixed bias the current was registered as a computer file. The range of the apply bias $V$ was from zero to $100 \mathrm{~V}$. Above $20.00 \mathrm{~V}$ and indicated by the first arrow, is the bias which marks the beginning of oscillatory behavior. For the bias region between both arrows, the sample presents bifurcations.

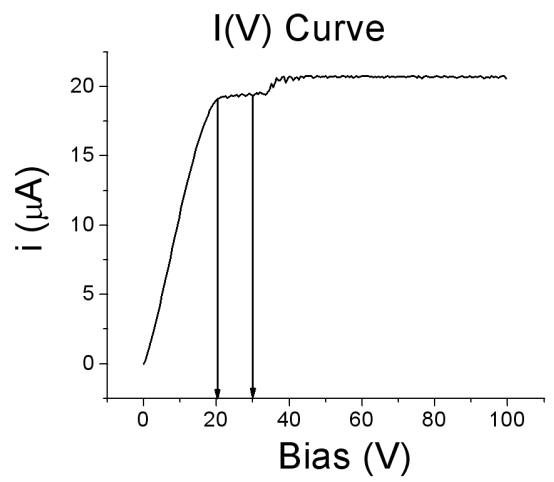

FIG. 1: I(V) characteristics for BH9816 grown at $215^{\circ} \mathrm{C}$.

We have measured LFO for applied bias $V$ between 20.00 and $30.00 \mathrm{~V}$. A typical LFO in chaotic behavior, measured at a temperature of $160 \mathrm{~K}$, under illumination with a constant current of $15 \mathrm{~mA}$ and fixed DC bias of $25.40 \mathrm{~V}$ is presented in Fig. 2. These oscillations are evidence of electric field domains being destroyed at the anode [9]. The magnitude order of LFO frequencies is of $10^{2} \mathrm{~Hz}$.

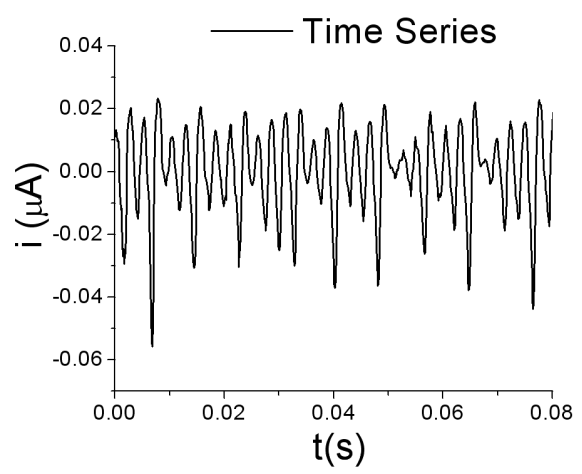

FIG. 2: Time series for chaotic behavior.

The Fig. 3 shows two bifurcation diagrams obtained from $\mathrm{I}(\mathrm{t})$ time series under applied bias $V$ in the domain of 20.00$26.00 \mathrm{~V}$ with step of $0.02 \mathrm{~V}$. Such diagrams were construed by minima sequence $\left\{S_{n}\right\}$, of each time series. In Fig. $3 b$, we present the bifurcation diagram after the application of the noise reduction algorithm in these time series. As result, the bifurcation diagram presents a clear region of period-4 cycles. Unfortunately one "arm" of the bifurcation diagram has vanished with this process.
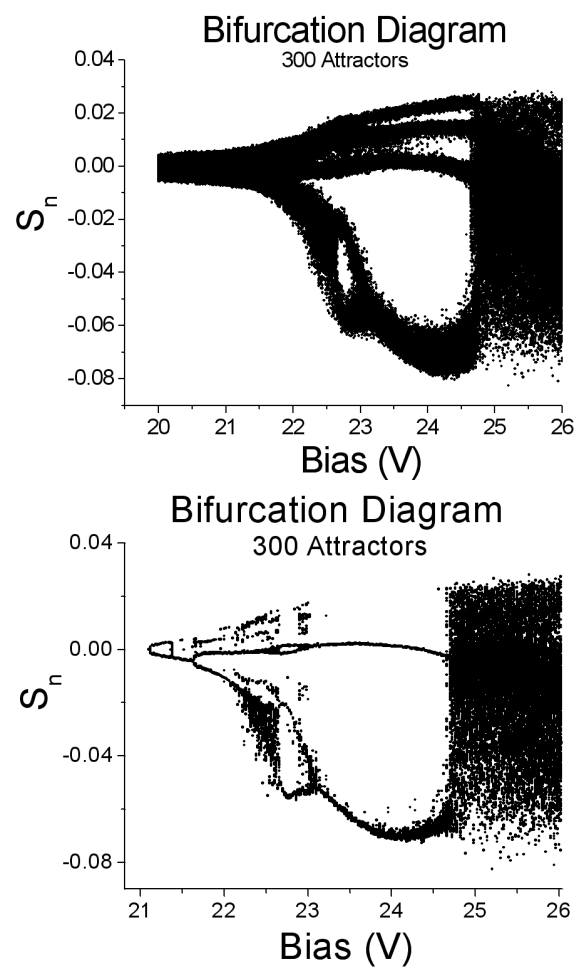

FIG. 3: Experimental bifurcation diagram of the current minima of the time series as a function of the applied bias in the sample. (a) The original bifurcation diagram, and (b) With noise reduction. Measurements were carried at $160 \mathrm{~K}$ and $\mathrm{I}_{L E D}=15 \mathrm{~mA}$.

The second sample grown at $265^{\circ} \mathrm{C}$ will be addressed as BH9817. Fig. 4 show the $\mathrm{I}(\mathrm{V})$ characteristics measured at $106 \mathrm{~K}$ under illumination with a constant current of $2 \mathrm{~mA}$ through the LED. The range of the applied bias $V$ was from zero to $100 \mathrm{~V}$. The region between arrows, defines the LFO measurements which we considered for the bifurcation diagram. At applied bias within the range of 60.00 to $82.00 \mathrm{~V}$ we observed high current oscillations, but that were very unstable and hard to register.

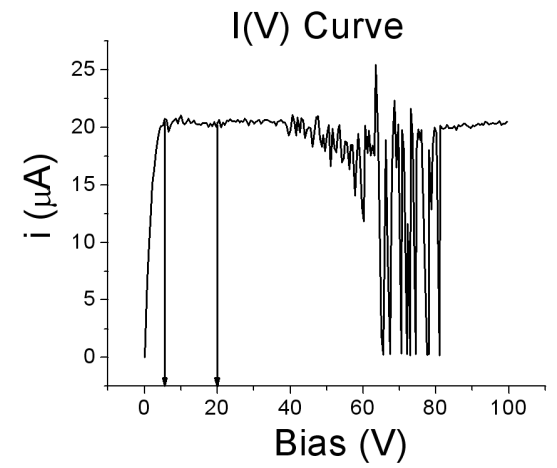

FIG. 4: I(V) characteristics for $\mathrm{BH} 9817$ grown at $265^{\circ} \mathrm{C}$. 
In Fig. 5, we have a bifurcation diagram obtained for time series $\mathrm{I}(\mathrm{t})$ under applied bias $V$ in the domain of $5.00-10.00 \mathrm{~V}$ with step of $0.01 \mathrm{~V}$. The measurement procedure was the same of the BH9816.

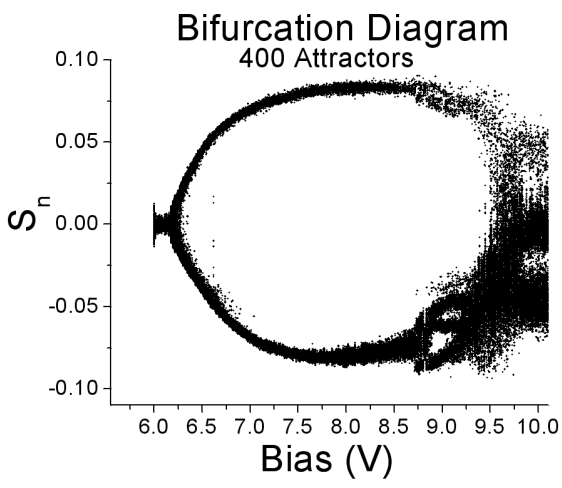

FIG. 5: Experimental bifurcation diagram for the minima of the current time series as a function of the applied bias. Measurements at $106 \mathrm{~K}$ with $\mathrm{I}_{L E D}=2 \mathrm{~mA}$. Noise reduction for this diagram was not considered due to signal deformation.

In this bifurcation diagram, we observe a more a new feature. Although hard to identify due to intrinsic noise, the most probable bifurcation sequence is: period-1, period-2, period-4 or period- 8 cycles followed by a probable chaos regime. For these data noise reduction algorithm was tested but proved to be inadequate since the signal/noise ratio was very high. As a consequence, the noise reduction algorithm removes part of real signal, resulting in a deformed bifurcation diagram.

\section{SUMMARY}

In summary, we measured $\mathrm{I}(\mathrm{V})$ and LFO I(t) for LT-MBE GaAs samples at controlled temperature and illumination conditions. Bifurcation diagrams were reconstructions from the minima sequence as a function of the applied bias. The noise reduction algorithm applied to the data of both BH9816 and BH9817 samples allowed the cleaning bifurcation, but, in both cases it was harmful to the time series. For the sample BH9817 which presented the worse the signal/noise ratio the noise reduction algorithm have not elucidated the blurred original bifurcation diagram. The bifurcation diagrams presented routes to chaos which resembles the Hopf type diagram with a bifurcation sequence of period-1-2-4 cycles and possible period- 8 cycles followed by a possible chaos. Finally, the reduction of periodicity in the bifurcation sequence of both samples might be an evidence of a complex dependence on the control parameter: temperature, illumination and applied DC bias $V$, of the experimental system. And the competition on g-r processes. We will discuss this in detail in another work which considers time series obtained from numerical integration of a differential equation system of a physical model of the relevant g-r mechanisms considered for the present work.

\section{Acknowledgements}

The authors would like to acknowledge the Brazilian agencies CNPq and FAPEMIG for financial support.
[1] R. M. Rubinger, R. L. da Silva, A. G. de Oliveira, G. M. Ribeiro, and H. A. Albuquerque, Chaos 13, 457 (2003).

[2] R. M. Rubinger, A. G. de Oliveira, J. C. Bezerra, G. M. Ribeiro, W. N. Rodrigues, and M. V. B. Moreira, Phys. Rev. B 62, 1859 (2000).

[3] G. N. Maracas, W. Porod, D. A. Johnson and K. Ferry, Physica B 134, 276 (1985).

[4] H. A. Albuquerque, A. G. de Oliveira, G. M. Ribeiro, R. L. da Silva, W. N. Rodrigues, M. V.B. Moreira, and R. M. Rubinger, J.
Appl. Phys. 93, 1647 (2003)

[5] H. D. I. Abarbanel, R. Brown, J. J. Sidorowich, and L. Sh. Tsimring, Rev. Mod. Phys. 65, 1331 (1993).

[6] R. Hegger, H. Kantz and T. Schreiber, Chaos 9, 413 (1999).

[7] R. L. da Silva, H. A. Albuquerque, R. M. Rubinger, A. G. de Oliveira, and G. M. Ribeiro, Physica D 194, 166 (2004).

[8] T. Schreiber, Phys. Rev. E 47, 2401 (1993).

[9] A. Neumann, J. Appl. Phys. 90 (1), 1 (2001). 